\title{
Regulierung hybrider Netzwerke im Schnittpunkt von Wirtschaft und Politik $^{1}$
}

\section{Irritationen im Recht: Kooperation zwischen staatlicher Verwaltung und Privaten in Netzwerkform}

Die heute zunehmend kooperativen Beziehungen zwischen Staat und Privaten zeichnen sich durch zahlreiche neue rechtliche Problemstellungen aus; es kann sogar behauptet werden, der neue Kooperatismus zwischen Staat und Privaten habe das Recht in eine tiefe Krise gestürzt: Denn indem dieser Kooperatismus einerseits die Verwaltung von den Fesseln der Gesetzesbindung befreit und andererseits Privaten Kompetenzen überträgt, die bisher der staatlichen Verwaltung zur Wahrung öffentlicher Interessen oblagen, werden die Grundpfeiler der rechtsstaatlichen Demokratie in Frage gestellt. Dies lässt sich anhand des Entscheids des schweizerischen Bundesgerichts 109 Ib 146 (1983) zur Vereinbarung über die Sorgfaltspflicht der schweizerischen Banken (VSB) mit aller Deutlichkeit zeigen. Aber nicht nur das: BGE 109 Ib 146 signalisiert auch, dass das Problem Kooperatismus eng mit einer anderen neuartigen Erscheinung verbunden sein kann, die das Recht in zunehmendem Maß irritiert: der Organisationsform des Netzwerks. ${ }^{2}$

Konkret ging es in BGE 109 Ib 146 darum, dass die Schweizerische Nationalbank, eine öffentlichrechtliche Anstalt des Bundes, ohne konkreten gesetzgeberischen Auftrag mit der überwiegenden Mehrheit der schweizerischen Banken in der Form bilateraler Verträge eine »Vereinbarung über die Sorgfaltspflichten bei Entgegennahme von Geldern « (VSB) erneuerte. ${ }^{3}$ Diese erneuerte VSB von 1982 enthielt nun verschärfte Regeln zur Offenlegung der Identität von Dritten, für deren Rechnung Vermögenswerte angelegt werden; im Gegensatz zur früheren Vereinbarung von 1977 wurde in dieser Version der VSB der Kreis derjenigen, die die Herkunft der anzulegenden Gelder nicht offen legen mussten, verkleinert. Der hiervon betroffene TreuhänderVerband verlangte von der Offenlegung der Identität ebenso befreit zu werden wie die Rechtsanwälte und die Treuhand- und Revisionskammer. ${ }^{4}$ Dies wies die Nationalbank

1 Ein evolutionstheoretischer Beitrag zur Morphogenese als koevolutorische Strategie sozialer Systeme, dargestellt anhand der Vereinbarung über die Sorgfaltspflicht der schweizerischen Banken. Ich danke Marc Amstutz, Werner de Capitani, Terrence Daintith, Vaios Karavas, Renate Schwob und Gunther Teubner für ihre weiterführenden Hinweise und Kommentare.

2 Zum Begriff des Netzwerks vgl. Gunther Teubner (2004) »Coincidentia oppositorum: Das Recht der Netzwerke jenseits von Vertrag und Organisation«, in: Marc Amstutz (Hg.) Die vernetzte Wirtschaft, Zürich: Schulthess, 11-42.

3 Erstmals mit der VSB vom 9. Dezember 1977. In BGE $109 \mathrm{Ib} 146$ ging es um die VSB vom 1. Juli 1982. Siehe den Vergleich zwischen den ersten zwei VSB-Versionen bei Peter Nobel (1987) »Die neuen Standesregeln zur Sorgfaltspflicht der Banken«, 39 Wirtschaft und Recht, 149-166, m.w.N.

4 Es ist anzunehmen, dass der Treuhänder-Verband mit der Beschwerde vor allem dem Image-Schaden begegnen wollte. Allerdings nahm tatsächlich die überwiegende Mehrheit der Banken an der VSB teil, so dass den Mitgliedern des Treuhänder-Verbands aus der Beschränkung an Vertragspartnern ein wirtschaftlicher Nachteil erwuchs. 
mit einem Schreiben an die Gesuchstellerin ab. Der Treuhänder-Verband focht dieses Schreiben als Verfügung mit Verwaltungsgerichtsbeschwerde beim Bundesgericht an. $^{5}$

An dieser Stelle ist auf die Vorgeschichte der VSB hinzuweisen: Ausländische Anleger hatten Mitte der siebziger Jahre Geld im Wert von über zwei Milliarden Franken mit Hilfe von Garantien und Bürgschaften einer schweizerischen Großbank unter Verstoß gegen schweizerische Kapitalzuflussregeln zur in der Schweiz tätigen TexonFinanzanstalt in Vaduz (Lichtenstein) transferiert. ${ }^{6}$ Das angelegte Geld wurde vor allem in Verbindung mit Steuerflucht und Schwarzgeld gebracht. ${ }^{7}$ Der ausgelöste - zunächst politikinterne, sodann aber auch wirtschaftliche - Skandal traf das Image des schweizerischen Bankensektors derart, dass in direkter Folge die Selbstbeschränkung durch die know your customer-Rule in der Form der Branchenvereinbarung VSB realisiert wurde. Die erste VSB aus dem Jahr 1977 verlangte in Art. 4 insbesondere die Ermittlung der Herkunft der Gelder. ${ }^{8}$ Nachdem der Texon-Skandal abgeklungen war, vereinbarten die Banken im Jahr 1982 mit der Nationalbank eine Neuauflage der VSB, die sich zwar immer noch gegen die Förderung der Wirtschaftskriminalität durch das Bankengeschäft richtete, ${ }^{9}$ jedoch die Abklärungspflichten weitgehend formalisierte. ${ }^{10}$

Das Bundesgericht trat nun auf die Beschwerde in BGE 109 Ib 146 mit der Begründung nicht ein, es handle sich bei der VSB um einen privatrechtlichen Vertrag und somit beim Schreiben der Nationalbank an den Treuhänder-Verband nicht um eine anfechtbare Verfügung. Denn die VSB bezwecke primär, Vorgaben von ausländischem Strafrecht umzusetzen, um Konflikte mit ausländischen Rechtsordnungen zu vermei-

5 BGE $109 \mathrm{Ib} 146,146 \mathrm{f}$.

6 Eine gute Übersicht über den Ablauf des Texon-Skandal gibt die NZZ-Berichterstattung: NZZ vom 26.4.77: 15 und NZZ vom 28.4.77: 13. Im Detail hierzu Joseph Jung (2000) From Schweizerische Kreditanstalt to Credit Suisse Corp. - The History of a Bank, Zürich: NZZ Buchverlag, 245 ff., vor allem 257. Vgl. auch BGE 105 Ib 348. Konkret lag ein Verstoß gegen Kapitalzuflussregeln vor, die den Zufluss ausländischer Gelder vor allem mit Hilfe von Abgaben verringern wollte.

7 Vor allem Italien war vom Kapitalabfluss ins Tessin betroffen: vgl. NZZ vom 20.4.77: 15, vom 26.4.77: 15, vom 28.4.77: 13 und vom 6.5.77: 17. Vgl. auch BGE $105 \mathrm{Ib} 348$, vor allem Sachverhalt A. sowie Erwägungen 10d) und 11; hierzu Georg Müller (1984) »Zur Rechtsnatur der Vereinbarung über die Sorgfaltspflichten der Banken bei der Entgegennahme von Geldern und über die Handhabung des Bankgeheimnisses «, 80 SJZ, 349-351, 350; Nobel (Fn. 2) 149 ff.; Peter Nobel (2004) Schweizerisches Finanzmarktrecht, Bern: Stämpfli, § 6 N 6 .

8 Der Kerngehalt der ersten VSB von 1977, mit der gebotenen Sorgfalt den wirtschaftlich Berechtigten festzustellen, ist bis heute nahezu unverändert geblieben: Art. 3 Abs. 1 VSB 2003. Vgl. zu den Anfängen der VSB Daniel Zuberbühler (1987) »Das Verhältnis zwischen der Bankenaufsicht, insbesondere der Überwachung der einwandfreien Geschäftstätigkeit, und der neuen Sorgfaltspflichtvereinbarung der Banken«, 39 Wirtschaft und Recht, 167-188 und Nobel (Fn. 3). Zu den aktuellen Entwicklungen u.a. Werner de Capitani (2004) »Die Aufsichtskommission VSB und das zehnte Gebot«, in: Hans Caspar von der Crone, Peter Forstmoser, Rolf Weber und Roger Zäch (Hg.) Aktuelle Fragen des Bank- und Finanzmarktrechts: Festschrift für Dieter Zobl zum 60. Geburtstag, Zürich: Schulthess, 63-70.

9 So explizit die Präambel der VSB 1982.

10 Art. 2-5 VSB 1982; siehe auch den Vergleich mit der VSB 1977 bei Nobel (Fn. 3). 
den (sic). Damit führe die Interessentheorie nicht zu einer Zuweisung zum öffentlichen Recht. Und nach der Subordinationstheorie zeige sich, dass die Nationalbank zwar zu ihrer Tätigkeit vom Bundesrat »ermuntert wurde«, aber keinen konkreten Gesetzgebungsauftrag umsetzte und es den einzelnen Banken demzufolge freigestanden habe, der Vereinbarung beizutreten. Dass eine vergleichbare Ordnung auch durch Gesetz hätte geschaffen werden können, sei nicht relevant. ${ }^{11}$ Allenfalls könne eine Aufsichtsbeschwerde gegen die Nationalbank geführt werden, denn die Nationalbank habe »in ihren privatrechtlichen Aktivitäten sinngemäss [sic] die verfassungsmässigen Grundrechte zu beachten ... Sie darf auch als Subjekt des Privatrechts insbesondere nicht rechtsungleich oder willkürlich Rechte erteilen oder Pflichten auferlegen. ${ }^{12}$

Die Rechtswissenschaft ist sich bis heute über die nötigen Anpassungen des Rechts an die offen zutage tretenden Tendenzen des Kooperatismus und der Privatisierung (oder Verwesentlichung der Staatsfunktionen ${ }^{13}$ ) uneinig: Auf der einen Seite wird die Ausdehnung des öffentlichen Rechts gefordert, ${ }^{14}$ worin allerdings die Gefahr besteht, die neuartigen kooperatistischen Formen unter den einschränkenden Regelungen zu ersticken. ${ }^{15}$ Auf der anderen Seite wird vorgeschlagen, das Recht solle die gesellschaftlichen Veränderungen durch die Verlagerung der entsprechenden Sachverhalte vom öffentlichen Recht ins Privatrecht und vor allem ins Vertragsrecht nachvollziehen $^{16}$, welches damit jedoch mit Vorgaben konfrontiert wird, die bisher im öffentlichen Recht angesiedelt waren, und somit in einem hohen Grad bis hin zur Infragestellung der eigenen Prämissen irritiert wird. ${ }^{17}$ Diese höchst voraussetzungsvolle Adaption des Rechts an den zunehmenden staatlichen Kooperatismus wird allerdings gleichzeitig weiteren Irritationen durch ebenso tiefgreifende und neuartige Umweltveränderungen ausgesetzt: den Umstellungen von markbezogenen Organisationsstrukturen auf der einen Seite und hierarchischen Organisationsstrukturen auf der anderen Seite (im Recht abgebildet durch Vertrag und Personenverbindungen) auf netzwerkartige Strukturen, auf die das Recht nicht mit eigenen Kategorien vorbereitet ist. Dies

11 BGE 109 Ib 146 (1983) 149 ff., vor allem E. 2 und 3.

12 BGE 109 Ib 146 (1983) 155, E. 4.

13 So etwa Georg Müller (2005) »Wege zu einem schlanken Staat: Überprüfung der Aufgaben stete Pflicht der Staatsleitung «, NZZ vom 16. März, 15.

14 Vgl. Paul Richli (1985) »Die verwaltungsrechtliche Rechtsprechung des BGer 1983: Bankengesetz«, 121 ZBJV, 428-430; vgl. jüngst auch Yvo Hangartner (2003) »Bemerkungen zu BGE 129 III 35«, AJP, 690-693.

15 Mit BGE 129 III 35 (2003) reagierte das Bundesgericht konsequent auf die von der Politik getriebene Staatsaufgabenverwesentlichung; hierzu Marc Amstutz, Andreas Abegg und Vaios Karavas (2006) Soziales Vertragsrecht, Basel, im Erscheinen.

16 So z.B. Arnold Marti (2002) »Aufgabenteilung zwischen Staat und Privaten auf dem Gebiet der Rechtsetzung - Ende des staatlichen Rechtsetzungsmonopols?«, AJP, 1154-1162, 1158.

17 Z.B. in BGE 129 III 35 (2003) treten verfassungmässige Aufgaben des Vertragsrechts offen zu Tage: Amstutz, Abegg und Karavas (Fn. 15). Zur Geschichte der Selbstregulierung im Bankensektor vgl. Yves Sancey (2004) Un capitalisme de Gentlemen. Emergence et consolidation de l'autorégulation bancaire en Suisse et en Angleterre (1914-1960), Lausanne: Thèses de la Faculté de SSP; Hugo Bänziger (1986) Die Entwicklung der Bankenaufsicht in der Schweiz seit dem 19. Jahrhundert, Bern: Haupt. 
zeigt sich deutlich, wenn man die weiteren Suchbewegungen der durch die VSB tangierten Gesellschaftssysteme verfolgt.

Nachdem das Bundesgericht zwar die VSB dem Privatrecht zugeschlagen und zugleich auf die »sinngemässe« Grundrechtsbindung der Nationalbank hingewiesen hatte, und nachdem die nachfolgende Kritik der Rechtswissenschaft auf die fehlende Stabilisierungsfähigkeit der vom Bundesgericht getroffenen Selektion hingewiesen hatte und die VSB ins öffentliche Recht zurückführen wollte, ${ }^{18}$ reagierten die Akteure in Wirtschaft und Politik: Für die neue Fassung der VSB im Jahr 1987 zog sich die Nationalbank aus der Vereinbarung zurück, womit im Resultat jenen Kritikern Recht gegeben wurde, die die fehlende gesetzliche Grundlage für die Beteiligung der Nationalbank an der VSB moniert hatten. ${ }^{19}$ Mit dieser Änderung sollte der Gefahr vorgebeugt werden, dass die VSB, wie es einige Kritiker verlangten, doch noch dem öffentlichen Recht zugeschlagen oder - noch unerwünschter - eine weitgehende Gesetzgebung auslösen würde. ${ }^{20}$ An die Stelle der Nationalbank trat die eidgenössische Bankenkommission (EBK), die über die geeigneten gesetzlichen Kompetenzen als Aufsichtsbehörde verfügte, sich auf die VSB einzulassen, ja sich sogar dazu berufen fühlte, im Jahr 1991 Teile der VSB teilweise außer Kraft zu setzen. ${ }^{21}$ Im Gegensatz zur Nationalbank trat zudem die EBK nicht mehr als direkter Vertragspartner der Banken auf, womit wohl ebenfalls einer weiteren Politisierung vorgebeugt werden sollte. Die Position als Zentrum des Netzwerks, das die einzelnen Verträge mit den Banken abschließt, übernahm nun der private Branchenverband der Banken, die Schweizerische Bankiervereinigung, während die EBK als Aufsichtbehörde fungierte. ${ }^{22}$

18 Hierzu vor allem Müller (Fn. 7); René Rhinow (1985) »Verfügung, Verwaltungsvertrag und privatrechtlicher Vertrag «, in: Juristische Fakultät der Universität Basel (Hg.) Privatrecht - Öffentliches Recht - Strafrecht: Grenzen und Grenzüberschreitungen; Festgabe zum Schweizerischen Juristentag, Basel: Helbing \& Lichtenhahn, 295-322; René Rhinow (1985) »Verwaltungsrechtlicher oder privatrechtlicher Vertrag: Fiskalwirkung der Grundrechte «, 3 recht, 57-64.

19 Dass die Nationalbank 1977 ins Spiel kam, wurde damit begründet, dass mit dem TexonSkandal nicht nur das Image des schweizerischen Bankenplatzes im Allgemeinen und die Aktien der Kreditanstalt im Speziellen unter Druck kamen, sondern auch der Schweizerfranken eine markante Abwertung erfuhr: NZZ vom 27.4.77: 17 und vom 5.5.77: 29.

20 So auch die Interpretation von Zuberbühler (Fn. 8) 167.

21 Konkret ging es um das Verbot der Formulare B durch das Rundschreiben der EBK vom 25. April/1. Juli 1991: Jahresbericht der EBK zum Jahr 1991, 17 f. Die Formulare B erlaubten den Treuhändern zu deklarierten, dass der wirtschaftliche Berechtigte bekannt sei, aber nicht offen gelegt werde.

22 Vgl. VSB 2003 vom 2.12.2002, abrufbar unter: http://www.swissbanking.org/1116_d.pdf (zuletzt besucht am 20.2.06). Die EBK erteilt Weisungen (Art. 3, N 34.4), erhält Verstösse gemeldet (Art. 10 und 12 Abs. 9) und genehmigt Änderungen der VSB (Art. 14 Abs. 3). Die Geldwäschereiverordnung der EBK, welche die VSB überlagert, wurde erstmals 1991 erlassen: hierzu die prägnante Darstellung bei de Capitani (Fn. 8) 64. Allerdings konnten diese Änderungen weiteren Druck (im Rahmen des >war on drugs $<$, >mani pulite < und jüngst $>$ Terrorismus $<$ ) auf den Bankenplatz Schweiz und auf die VSB nicht verhindern. Vgl. u.a. Bundesblatt 1989 II 1061 und 1089 f., Bundesblatt 1993 III 277, Bundesblatt 1996 III 1101. Zur Terrorismusbekämpfung vgl. Art. 1 der VSB 2003. 
Wie reagierte das Bundesgericht auf diese Entwicklungen, d. h. auf die Kritik der Wissenschaft an BGE $109 \mathrm{Ib} 146$ und auf die Anpassungsbewegungen von Politik und Wirtschaft? Bereits zwei Jahre nach BGE $109 \mathrm{Ib} 146$ hatte das Bundesgericht erneut die Möglichkeit, sich zur VSB zu äußern. Obwohl der Fall ganz anders lag, meinte das Bundesgericht in direkter Anspielung auf das vorgehende Urteil 109 Ib 146, es könne offen bleiben, ob die VSB privatrechtlicher oder öffentlichrechtlicher Natur sei; auf jeden Fall binde sie die EBK weder bei der Auslegung des Bankengesetzes noch in den Aufgaben als Aufsichtsinstanz. ${ }^{23}$ Mit dem Entscheid BGE 125 IV 139 (1999) relativierte das Bundesgericht ein weiteres Mal die Bedeutung der VSB ebenso wie die dadurch konstituierte Selbstregulierung des Bankensektors. ${ }^{24}$ In der Rechtswissenschaft bleibt bis heute umstritten, ob die VSB zum Privatrecht oder öffentlichen Recht zuzuweisen ist. ${ }^{25}$

In der Folge will ich mit einem Blick auf die Nachbarwissenschaften die These verfolgen, dass die Rechtswissenschaft zwar den neuartigen Kooperatismus zwischen Staat und Privaten als Ausgangspunkt der anhaltenden Irritationen erkannt hatte, aber auf diese neuartigen Irritationen, die sich im Schnittpunkt einer Diskurskollision babylonischen Ausmaßes befindet, mit den alten starren Konzepten nicht angemessen reagieren konnte und zudem die mit dem Kooperatismus verschlaufte Emergenz der netzwerkartigen Organisation vernachlässigte. Folglich braucht es eine Theorie, die die dynamische Koevolution der verschiedenen beteiligten Diskurse thematisieren kann. Ich werde hierzu die Evolutionstheorie einsetzen und dabei versuchen, das naturwissenschaftliche Konzept der Morphogenese fruchtbar zu machen.

\section{Die Morphogenese hybrider Netzwerke}

Nach Kämper/Schmidt geht es bei Netzwerken nicht um die Frage der Abstimmung von Funktionssystemen oder gar um eine Integration der Gesellschaft. ${ }^{26}$ Meine Analyse der bisherigen koevolutorischen Suchbewegungen rund um das VSB-Netzwerk deuten jedoch gerade in die entgegengesetzte Richtung: Das Netzwerk VSB und ganz

23 BGE $111 \mathrm{Ib} 127,127 \mathrm{f}$.

24 Für die Auslegung der strafrechtlichen Norm von Art. 305ter StGB (Geldwäscherei) bilde die VSB lediglich eine Auslegungshilfe: BGE 125 IV 139, E. 3d, 144 f.

25 Für die Zuweisung zum Privatrecht: Marti (Fn. 16) 1158 f. und Arnold Marti (2000) »Selbstregulierung anstelle staatlicher Gesetzgebung?«, Schweizerisches Zentralblatt für Staatsund Verwaltungsrecht (ZBL), 561-586, 576. Für die Zuweisung zum öffentlichen Recht: Ulrich Häfelin und Georg Müller (2002) Allgemeines Verwaltungsrecht, Zürich, N 1060. Für eine Zuweisung zum Privatrecht unter Bewahrung der Grundrechtsbindung: Markus Schefer (2002) »Grundrechtliche Schutzpflichten und die Auslagerung staatlicher Aufgaben «, AJP, 1131-1143, 1139 f. Für die dogmatische Darstellung der VSB sei auf die einschlägige Literatur verwiesen, etwa Nobel (Fn. 7) § 6 mit ausführlichen Literaturhinweisen.

26 Eckard Kämper und Johannes Schmidt (2000) »Netzwerke als strukturelle Kopplung«, in: Johannes Weyer (Hg.) Soziale Netzwerke: Konzepte und Methoden der sozialwissenschaftlichen Netzwerkforschung, München: Oldenbourg, 211-236, 227; a.A. Dirk Messner (1997) »Netzwerktheorien: Die Suche nach Ursachen und Auswegen aus der Krise staatlicher Steuerungspolitik«, in: Elmar Altvater, Achim Brunnengräber, Markus Haake und Heike Walk (Hg.) Vernetzt und verstrickt: Nicht-Regierung-Organisationen als gesellschaftliche Produktivkraft, Münster: Westfälisches Dampfboot, 27-64, 42 f. 
allgemein hybride Netzwerke, die zwischen sozialen Subsystemen der Gesellschaft positioniert sind, können Strategien dieser sozialen Subsysteme darstellen, um gleichzeitig ihre spezifisch systemische Eigenart zu erhalten und auf widersprüchliche Irritationen so weit als nötig zu reagieren. ${ }^{27}$ Damit geht es um nichts weniger als um die $s o$ zialintegrative Funktion der Evolution. Das bedarf weiterer Erläuterung:

Verschiedentlich wurde darauf hingewiesen, dass hybride Netzwerke eine institutionelle Reaktion auf ambivalente, widersprüchliche oder paradoxe Anforderungen sind: Da den gesteigerten Anforderungen aus der Umwelt nicht zu entkommen ist, nähert sich das System an diesem Ort der Komplexität der Umwelt. ${ }^{28}$ Dieser Vorgang, in welchem das System seine innere Konsistenz in einem Teilbereich senkt, kann in Anlehnung an die Naturwissenschaften als Morphogenese bezeichnet werden. ${ }^{29}$ In der Tat reagiert das Netzwerk VSB, wie sogleich detaillierter zu beschreiben sein wird, gleich auf mindestens drei paradoxe Anforderungen der Umwelt, die sich aus dem Bankenskandal und dem (für das Wirtschaftssystem freilich unbequemen) Ruf nach Einschränkung der wirtschaftlichen Logik ergeben. Zunächst möchte ich jedoch auf das von den Naturwissenschaften in den letzten Jahren weiterentwickelte Konzept der Morphogenese eingehen, das mir als Metapher (d. h. als inspirierende Lösungsskizze) zur Beschreibung der Funktion hybrider Netzwerken in der Evolution sozialer Systeme dienen soll.

Die neueren naturwissenschaftlichen Theorien zur Morphogenese passen erstaunlich präzise zum kauffmanschen Konzept der spontanen Ordnung, das Amstutz für die Rechtstheorie fruchtbar gemacht hat. Wie S.A. Kauffman gezeigt hat, erfolgt die Evolution eines Systems nicht nur durch die Selektion von geeigneten Variationen, sondern die Selektion muss mit einer inneren so genannt spontanen Ordnung zusammentreffen, die die Evolution eines Systems überhaupt erst ermöglicht. ${ }^{30}$ Diese spontane Ordnung hält das System in einer Konsistenz, in der es am besten auf die evolutionären Prozesse vorbereitet ist. Kauffman hat belegt, dass es Systemen dann möglich ist, auf Irritationen von Außen zu reagieren, wenn ihre epistatische Konnektivität $\mathrm{K}=2$ ist. ${ }^{31}$ Ein Kristall mit $\mathrm{K}=1$ ist dagegen gegenüber externen Irritationen unempfäng-

27 Vgl. Marc Amstutz (2001) Evolutorisches Wirtschaftsrecht: Vorstudien zum Recht und seiner Methode in den Diskurskollisionen der Marktgesellschaft, Baden-Baden: Nomos, 290 ff.; Gunther Teubner (2004) Netzwerk als Vertragsverbund: Virtuelle Unternehmen, Franchising, Just-in-time in sozialwissenschaftlicher und juristischer Sicht, Baden-Baden: Nomos, 29.

28 Gunther Teubner (2001) »Das Recht hybrider Netzwerke«, 165 ZHR, 550-575; Karl-Heinz Ladeur (1995) Das Umweltrecht der Wissensgesellschaft: von der Gefahrenabwehr zum Risikomanagement, Berlin, $31 \mathrm{ff}$.

29 Für die Naturwissenschaften vgl. Alexander Spirov (1998) »Game of Morphogenesis: What Can We Learn from the Pattern-Form Interplay Models?«, 8 International Journal of Bifurcations and Chaos, 991-1001, m.w.N. Für die Sozialwissenschaften siehe Teubner (Fn. 28) sowie Niklas Luhmann (1987) Soziale Systeme: Grundriss einer allgemeinen Theorie, Frankfurt a.M.: Suhrkamp, 480 f. und 485 f. m.w.N. zur Rezeption des Begriffs in den Sozialwissenschaften.

30 Stuart Kauffman (1993) The origins of order, self-organization and selection in evolution, New York: Oxford University; ders. (2000) Investigations, Oxford: Oxford University.

31 In der Biologie bezeichnet die epistatische Konnektivität die Abhängigkeit zwischen Genen, d.h. dass das eine Gen die Auswirkungen eines anderen Gens »verdeckt«. Wenn also die Variante a den Phänotyp A und die Variante b den Phänotyp B auslöst, jedoch die Kombination ab allein im Phänotyp A resultiert, dann ist a epistatisch zu b. 
lich, und bei einer hohen Konnektivität von $\mathrm{K}=\mathrm{N}-1$ driftet das System quasi bei jedem Schmetterlingsschlag ins Chaos ab. Vereinfacht gesagt: Die Elemente des Systems müssen in dem Grad lose verknüpft sein, dass sie die Irritationen »am Rande des Chaos « aufnehmen können, jedoch nur gerade so lose, dass sich das System trotz Irritationen als solches erhält. ${ }^{32}$ Der Ort im Sinne der »unit of selection «, an welchem die Evolution unter Erhaltung der Eigenrationalität erst ermöglicht wird, ist nicht das System als Ganzes. Dieses könnte die Anpassungsleistung nicht erfüllen. Vielmehr differenziert das System bestimmte Bereiche aus, die diese Anpassungsleistung erbringen müssen und hierfür mit der entsprechenden Umwelt gekoppelt sind. ${ }^{33}$

An dieses Konzept der spontanen Ordnung schließen nun die neueren Theorien der Morphogenese an: Nach Harrison bezeichnet die Morphogenese die Entstehung eines komplexen Systems aus einem einfacheren System, wobei dieser Prozess innere spontane Ordnung voraussetzt. ${ }^{34}$ Eine zentrale Stellung im Prozess der Morphogenese nehmen die Interaktionen zwischen den dynamischen Elementen des Systems ein: Einerseits müssen Veränderungen des Systems in Relation zu seiner Umwelt schnell und präzis erfasst sowie im System kommuniziert werden, andererseits sollen in umgekehrter Richtung Veränderung des Systems ausgelöst werden. ${ }^{35}$ Bei Zellen besteht das Zusammenspiel zwischen spontaner Ordnung und Formveränderung aus einem komplexen bio-mechanischen und chemischen Prozess, in welchem - in nuce - in einem ersten Schritt die Zelle ihre Position (Form) in ihrer Umwelt und insoweit auch die Umwelt in biomechanischer Weise »abtastet«, sodann diese Information an das Genom im Zellkern gesandt wird und schließlich das Genom eine Genkaskade freisetzt, um weitere Änderungen auf dem angestrebten Weg der Morphogenese auszulösen. Vereinfacht gesagt werden also morphogene Änderungen ausgelöst, diese sodann auf die angestrebte Position im System und die angestrebte Position relativ zur Umwelt des Systems überprüft sowie in einer Feedbackschlaufe mit den angestrebten Änderungen abgeglichen, entsprechend diesem Resultat und der angestrebten Morphogenese wiederum neue Änderungen ausgelöst usw. ${ }^{36}$ Diese morphogene Schlaufe ist

32 Kauffman (1993) (Fn. 30) 219 ff. und 255 ff.; hierzu Amstutz (Fn. 27) 278 ff., 286 ff. und 290 ff.; vgl. auch Alan Watson (2001) The Evolution of Western Private Law, Baltimore: John Hopkins University, $264 \mathrm{f}$.

33 Niklas Luhmann (1993) Das Recht der Gesellschaft, Frankfurt a.M.: Suhrkamp, 281; so auch Amstutz (Fn. 27) 290 ff. und Watson (Fn. 32) 264.

34 Lionel Harrison (1981) »Physical chemistry of biological morphogenesis«, 10 Chemical Society Reviews, 491-528, 491 und 502; David Depew und Bruce Weber (1997) Darwinism Evolving: Systems Dynamics and the Genealogy of Natural Selection, Cambridge, Mass.: MIT, 528 f., m.w.N.

35 Frederick Cummings (1994) »Aspects of growth and form«, 79 Physica D: Nonlinear Phenomena, 146-163; Spirov (Fn. 29).

36 Natalie Bjorklund und Richard Gordon (1993) »Nuclear state splitting: a working model for the mechanochemical coupling of differentiation waves to master genes«, 24 Russian J. Dev. Biol., 79-95; ähnlich bereits Harrison (Fn. 34) 502 ff. und Lionel Harrison (1988) »Kinetic Theory of Living Pattern and Form and Its Possible Relationship to Evolution«, in: Bruce Weber, David Depew und James Smith (Hg.) Entropy, Information, and Evolution: New Perspectives on Physical and Biological Evolution, Cambridge, Mass.: MIT, 5374, 63 und 73, mit Bezug auf S.A. Kauffman. 
allerdings nur eine Quelle der Veränderung. Ebenso maßgeblich sind die bereits erwähnte spontane Ordnung sowie die rigiden geometrischen Zwänge (Constraints), denen das System unterliegt und entlang welchen das System evolviert. ${ }^{37}$

Will man dieses Bild der Morphogenese auf den Fall hybrider Netzwerke anwenden, kann daraus geschlossen werden, dass ein System - in casu VSB vor allem die Wirtschaft, aber auch die Politik - dann eine besonders flexible Teilbereichsordnung ausdifferenziert, wenn es auf intensive und komplexe Irritationen aus der Umwelt mit Kohärenzsenkung reagieren muss, d. h. einen Prozess hin zu einer komplexeren Form vollzieht - selbstverständlich unter gleichzeitiger Bewahrung der eigenen Autopoiese. Während die teilbereichsspezifische Rationalität für eine gewisse Evolutionsresistenz (Form) sorgt, werden die dynamischen Elemente der Teilbereichsordnung in dem Grad lose verknüpft, als es auf Irritationen aus der Umwelt zu reagieren gilt - aber auch nur so lose, dass jederzeit die teilbereichsspezifische Rationalität eingehalten werden kann. Die Form des hybriden Netzwerks mit ihren besonders lose verknüpften Elementen bietet sich nun - wie Teubner gezeigt hat - dort in besonderem Maß an, wo aus widersprüchlichen oder gar paradoxen Irritationen mit hoher Intensität interne Variationen gebildet werden sollen. ${ }^{38}$ Im Effekt geht es somit für das System darum, mithilfe des Netzwerks die innersystemische Relation von Funktion, Leistung und Reflexion derart neu auszurichten, dass es möglichst optimal auf intensive, komplexe und gar widersprüchliche Umweltanforderungen reagieren kann, ohne seine Eigenrationalität zu gefährden.

Diese Anpassung des Systems erfolgt nun in einer morphogenen Feedbackschlaufe entlang der systemeigenen Constraints: An den Orten der strukturellen Kopplungen tastet das System seine Form und seine Position in der Umwelt ab, und aus diesen Informationen gewinnt das System im Rahmen der teilbereichsspezifischen Rationalität Variationen, die einer Selektion (Änderung oder Nichtänderung der bisherigen Elemente und Strukturen) zugeführt werden. Die durch die Selektion ausgelöste Modifikation ebenso wie die Ablehnung der Modifikation werden wiederum auf die Position im System und - erneut mithilfe struktureller Kopplungen - die Position in der Umwelt abgetastet, was nun in einer weiteren Schlaufe neue Variationen und Selektionen auslösen kann. Das Besondere an der Form des hybriden Netzwerks ist nun, dass es gleichzeitig eine Vielzahl struktureller Kopplungen mit verschiedenen Systemen in Anspruch nehmen kann. Damit gewinnt die morphogene Schlaufe an Bedeutung, denn es werden die Resultate bisheriger Selektionen mit den internen und externen Anforderungen an das System abgeglichen und allenfalls Korrekturen veranlasst, bis on the long run ein punktuelles Equilibrium zwischen dem System und seiner Umwelt erreicht ist. In dieser Optik ist die morphogene Schlaufe Voraussetzung dafür, dass sich ein System unter Bewahrung der Eigenrationalität auf intensive Anforderungen der Umweltsysteme einlassen kann und ein punktuelles Equilibrium mit seinen Umweltsystemen anstreben kann.

37 Frederick Cummings (2001) »The Interaction of Surface Geometry with Morphogens«, 212 Journal of Theoretical Biology, 303-313.

38 Gunther Teubner (1989) Recht als autopoietisches System, Frankfurt a.M.: Suhrkamp, 75; Amstutz (Fn. 27) 290 ff. 
Ich will nun am konkreten Beispiel des VSB-Netzwerks darstellen, dass in casu die Wirtschaft auf intensive und widersprüchliche Irritationen mit der Form des hybriden Netzwerks reagierte und damit eine spezifische Teilbereichsordnung ausdifferenzierte, die für die Wirtschaft die Bedingungen der Möglichkeit von Koevolution mit den Umweltsystemen durch morphogene Schlaufen herstellte. Auf der Grundlage des Modells einer polykontexturalen Gesellschaft ${ }^{39}$ will ich zudem versuchen, normative Schlüsse aus der mit der Evolutionstheorie gewonnenen Beobachtung zu ziehen. Dabei gilt es allerdings die rechtseigenen Constraints in der Form der rigiden Anschlusszwänge der Rechtsdogmatik zu berücksichtigen. Die Institute Organisation und Vertrag bauen im Recht auf einer langen Tradition auf, während die Formen zwischen Organisation und Vertrag und zwischen Staat und privater Selbstorganisation mit der Emergenz des Absolutismus aus dem Recht verbannt wurden. ${ }^{40}$ Es steht somit kein aktualisierter, d. h. an die heutige Rechtsdogmatik ohne weiteres anschließbarer Pool an Elementen zur Verfügung, aus welchem die für die neuartigen Phänomene adäquate Variationen ausgewählt werden könnten. Zudem können vorliberale Lösungen einer stratifizierten Gesellschaft nur mit großer Vorsicht für eine postmoderne polykontexturale Gesellschaft des Rechts Berücksichtigung finden. Die Positionierung neuer Phänomene wie hybride Netzwerke zwischen staatlicher Verwaltung und Privaten kann somit nicht an bestehende Konzepte anschließen, und ein entsprechendes Rechtsgebiet aus dem Nichts zu erschaffen, ist schon aus Gründen der inneren Constraints der Rechtsdogmatik ausgeschlossen. Zu rigide wacht die Dogmatik über die Konsistenz neuer Normvariationen mit den bestehenden Normen. Folglich müssen die neuartigen Erscheinungen, die sich zwischen den alten Dichotomien Vertrag und Organisation sowie Staat und privater Selbstorganisation situieren, in diesen bestehenden Dichotomien auf der einen Seite Aufnahme finden, wobei allerdings ihre »Kehrseite« auf der anderen Seite zu berücksichtigen ist.

\section{Die VSB im Licht einer evolutorischen Netzwerktheorie}

\section{Entfaltung paradoxer Anforderung: die Kooperation der Konkurrenten}

Der Texon-Skandal kann durchaus auch als innenpolitischer Skandal bezeichnet werden. Denn die schweizerische Politik, die durch Steuern und Abgaben vom Bankenge-

39 Hierzu u.a Gunther Teubner (1998) »Vertragswelten: Das Recht in der Fragmentierung von private governance regimes«, 17 Rechtshistorisches Journal, 234-265, 234 ff., vor allem $241 \mathrm{ff}$.

40 Typisch hierfür: Johann Heinrich Gottlob von Justi (1782/1756) Grundsätze der Polizeiwissenschaft, Göttingen: Vandenhoeck, Vorwort; Johann Heinrich Gottlob von Justi (1760) Die Grundfeste zu der Macht und Glückseeligkeit der Staaten, oder, Ausführliche Vorstellung der gesamten Policey-Wissenschaft, Königsberg: Hartung, Band 2, § 554-555. Zumeist ging es seit dem 16. Jahrhundert darum, die auf Kirche, Grundeigentümer, etc. zersplitterte Gerichtsbarkeit als einzige Art der Lokalverwaltung unter staatliche Kontrolle zu bekommen. Je mehr dies gelang, desto mehr deckten sich die Begriffe von politischer Macht (als Fähigkeit, Gehorsam zu erhalten) und Rechtsmacht in der Souveränität als hoheitliche Gewalt. hierzu Luhmann (Fn. 33) 407 ff. 
schäft profitiert, fürchtete noch mehr als bisher in Verruf zu geraten und fühlte sich entsprechend unter Druck, auf die Banken korrigierend einzuwirken. ${ }^{41}$ Allerdings fehlten der Politik aus mannigfaltigen Gründen wie Komplexität und Technizität, Mittelbegrenzung und Privatisierung sowie Globalisierung sowohl das Wissen wie auch die Mittel, um den dynamischen Wirtschaftssektor der Banken inner geeigneter Frist autoritativ zu regulieren. ${ }^{42}$ Ebenso war es das primäre Ziel der schweizerischen Banken, eine politische Regulierung zu vermeiden, die das »normale Bankgeschäft« erschwert hätte. ${ }^{43}$ Wie konnten die Banken dieses Ziel erreichen? Um ein unbequemes Korsett durch politische Regulierung zu vermeiden, musste die Politik von der Wirtschaft derart irritiert werden, dass für die Politik die Voraussetzungen, regulatorisch tätig zu werden, wegfielen. Wenn nun einzelne Wirtschaftsteilnehmer politische Vorgaben als politisch-ethische Richtlinien aus freien Stücken unilateral rezipiert hätten und so ihre wirtschaftlichen Möglichkeiten im Sinne der Politik beschränkt hätten, wären freilich für diese einzelnen Banken beachtliche Wettbewerbsnachteile gegenüber unfügsamen Konkurrenten entstanden - ohne dass damit das Problem für die Politik notwendigerweise aus der Welt geschafft worden wäre. Eine unilaterale Anpassung der Banken an die Anforderungen der Politik erwies sich folglich bereits aus systemimmanenten Constraints der Wirtschaft als illusionär. ${ }^{44}$

Die ordoliberale Theorie hat bekanntlich mit der Erkenntnis, dass eine Marktordnung nicht Folge, sondern Voraussetzung des Marktmechanismus ist, den Liberalismus vom Kopf auf die Füße gestellt. ${ }^{45}$ In diesem Sinn der ordoliberalen Theorie blieb den Banken, gemeinsam eine Marktordnung zu schaffen, die die wirtschaftlichen Möglichkeiten der wichtigsten Player im Bankensektor gleichzeitig und gleichmäßig einschränkt und damit zumindest im Kreis der wichtigen Marktteilnehmer im Effekt weitgehend wettbewerbsneutral ist, d. h. die neuen Sorgfaltspflichten kartellisiert. ${ }^{46}$ Ein entsprechendes gemeinsames Vorgehen der Konkurrenten hätte jedoch beachtliche Gefahren mit sich gebracht. Mit der Gründung einer formellen Organisation wären

41 Vgl. NZZ vom 6.5.77: 17 und NZZ vom 5.5.77: 25 und 29, vor allem die Erklärung des Bundesrates zum Bankskandal; Jung (Fn. 6) 261.

42 Vgl. u.a. Thomas Müller (1997) Verwaltungsverträge im Spannungsfeld von Recht, Politik und Wirtschaft: eine systemtheoretische Analyse von Verträgen zwischen dem Gemeinwesen und Privaten mit Hinweisen auf die rechtsdogmatischen Konsequenzen, Basel: Helbing \& Lichtenhahn, 142 ff.; Peter Uebersax (2001)»Privatisierung der Verwaltung «, ZBL, 393422.

43 Das genannte Ziel noch heute in bemerkenswerter Klarheit in der VSB formuliert: Ziff. 3 zu Art. 1 VSB 2003. Vgl. den Leitartikel der NZZ vom 7./.8.5.77 sowie die Berichte zur Debatte des Nationalrats in der NZZ vom 5.5.77: 25 und 29.

$44 \mathrm{Zu}$ den Problemen der Wirtschaftsethik vgl. Niklas Luhmann (1989) Die Wirtschaft der Gesellschaft, Frankfurt a.M.: Suhrkamp, 84 ff.

45 Vgl. die herausragende Darstellung der Zusammenhänge bei Rudolf Wiethölter (1968/ 1986) Rechtswissenschaft, Basel: Helbing \& Lichtenhahn; grundlegend hierzu Franz Böhm (1933) Wettbewerb und Monopolkampf, Berlin: Heymann.

46 Zum Zusammenspiel zwischen staatlicher Regulierung und Selbstregulierung aus Sicht der ordoliberalen Theorie vgl. Franz Böhm (1966) »Privatrechtsgesellschaft und Marktwirtschaft«, 17 ORDO, 75-151. Zum Kartellisierungseffekt sozialer Normen vgl. Stefan Huf (1999) »Die sozialstaatliche Einbettung der Marktökonomie«, 45 ZfS, 221-241, $227 \mathrm{f}$. 
sich die Konkurrenten derart nahe gekommen, dass sie die fundamentale Prämisse des Marktes in Frage gestellt und zudem entsprechende Reaktionen von Politik und Recht riskiert hätten. Der Bankensektor sah sich somit der paradoxen Anforderung ausgesetzt, unter Aufrechterhaltung der Konkurrenz zu kooperieren.

Die Form des Netzwerks löst solche Widersprüchlichkeit nicht - wie es vom Kartellrecht ${ }^{47}$ nahe gelegt wird - durch die Verdrängung der einen Seite, der Organisation, auf, sondern sie verwirklicht ein Sowohl-als-auch, respektive entfaltet das Paradox. Wie stellt sich dies in casu VSB dar? Im VSB-Netzwerk wurden Regelungen ausformuliert, mit welchen die Wirtschaft den Umweltanforderungen bis zu einem bestimmten Grad Rechnung trug - konkret im Bereich des Bankenwesens die Möglichkeiten der wirtschaftlichen Betätigung einschränkte. Indem nun diese Beschränkung der wirtschaftlichen Rationalität in bilateralen Arrangements, die sich wechselseitig voraussetzen, durch Vertrag auf Dauer stabilisiert wurde, resultierte ab einer bestimmten Schwelle von versammelter Marktmacht eine sogenannte Kartellisierung, d. h. die in den Verträgen stabilisierte Regelung wurde wettbewerbsneutral, da der eine Marktteilnehmer den anderen Marktteilnehmer nicht mehr durch Unterbietung des festgelegten Standards konkurrenzierte. Das Netzwerk erlangte somit in struktureller Kopplung zum Rechtssystem durch die jeweils bilateralen aber immer gleichen und aufeinander bezogenen vertraglichen Arrangements Kontrolle über das opportunistische Verhalten ihrer Teilnehmer in einem Teilbereich des Wettbewerbs, während sich die Netzwerkteilnehmer in den übrigen Bereichen weiter konkurrenzierten. ${ }^{48}$ Damit konnte einerseits jene Kooperation erreicht werden, die den politischen Forderungen entsprechen konnte, und andererseits war diese Kooperation in Form des Vertragsschlusses der einzelnen Bank mit einem neutralen Netzwerkzentrum derart lose und sachlich beschränkt, dass die Form der Konkurrenz nicht nur für das Wirtschaftssystem aufrecht erhalten wurde, sondern dies auch entsprechend an die anderen Systeme, vor allem an das Rechtsystem signalisiert werden konnte. ${ }^{49}$ Damit wurde gleichzeitig die Angriffsfläche für politisch-rechtliche Interventionen minimiert und somit einer übermäßigen Einflussnahme durch die gesetzgebende Politik vorgebeugt; wie das Netzwerk angelegt wurde und wie es vom Bundesgericht denn auch wahrgenommen wurde, sollte es

47 Das erste schweizerische Kartellgesetz mit dem Ziel des Wettbewerbsschutzes stammt freilich erst von 1985 (Amtliche Sammlung 1986, 874).

48 Abgesichert wurde dies durch die Schiedsklausel, die ein vertragliches Arrangement voraussetzte: Art. 14 VSB 1977.

49 Die zentrale Funktion des Vertrages für Netzwerke wird auch betont von Jörg Sydow und Arnold Windeler (2000) »Steuerung von und in Netzwerken - Perspektiven, Konzepte, vor allem aber offene Fragen«, in: idem (Hg.) Steuerung von Netzwerken, Opladen: Westdeutscher, 1-24, $14 \mathrm{f}$. Dabei handelt es sich jedoch weder um den klassischen Vertrag, noch um einen langfristigen Dauervertrag, sondern um einen mittelfristig angelegten Vertrag, der den Parteien eine massgebliche Freiheit in der Anpassung der Vertragsbeziehung an die sich wandelnden Umstände gibt: vgl. Oliver Williamson (1991) »Comparativ economic organization: The analysis of discrete structural alternatives «, in: Siegwart Lindenberg und Hein Schreuder (Hg.) Interdisciplinary Perspectives on Organization Studies, New York: Pergamon, 3-37. 
für das Recht nur ein lose verknüpftes Bündel von privatrechtlichen Verträgen darstellen.

Wie ist nun dieser Re-entry der Organisation in den Vertrag rechtlich einzuordnen? Vertragsrechtlich kann er problemlos in den (allenfalls normativ ausgelegten) Vertragsinhalt fließen ${ }^{50}$ Bei einem nüchternen Blick ins Kartellrecht kommen allerdings Zweifel auf, ob die VSB nicht vom Kartellgesetz erfasst werden müsste. ${ }^{51}$ Sieht man jedoch die VSB als Strategie der Wirtschaft, den Teilbereich des Bankenwesens so zu organisieren, dass dieser komplexe und intensive Irritationen der Umwelt aufgrund innerer Ordnung (im Sinne von S.A. Kauffman) absorbieren und darauf in flexibler Weise (im Sinne morphogener Schlaufen) reagieren kann, wäre es vor dem Hintergrund des Modells einer polykontexturalen Gesellschaft geradezu fatal, das Netzwerk vom Recht in die traditionellen Dichotomien von Konkurrenz versus Kooperation respektive Vertrag versus Organisation zu zwingen, das heißt vor allem als unzulässige Wettbewerbsabrede zu qualifizieren und damit das Element der Organisation zu unterdrücken oder aber als Organisation zu rekonstruieren, womit die zentrale Rolle des Vertrags unterbelichtet bliebe. Aus dieser Optik scheint gerade im Gegenteil der sogenannte Re-entry der Organisation in die Vertragsform schützenswert - zumindest soweit dieser dazu dient, dass die Wirtschaft Irritationen aus der Umwelt besser absorbieren und ihre Struktur entsprechend besser auf die Anforderungen der Umweltsysteme ausrichten kann. ${ }^{52}$

Indes dient das Netzwerk der Wirtschaft nicht allein dazu, intensive widersprüchliche oder gar paradoxe Anforderungen absorbieren zu können, sondern kann ihm darüber hinaus eine besondere sozialintegrative Funktion zukommen. Worin diese sozialintegrative Funktion des Netzwerks VSB liegt, und welche Rolle die erwähnten morphogenen Schlaufen spielen, will ich nun in der Folge erläutern.

\section{Kompatibilisierung widersprüchlicher Rationalitäten}

Nach dem Texon-Skandal musste der Bankensektor nicht nur eine Marktordnung bei gleichzeitiger Aufrechterhaltung der Konkurrenz hervorbringen, sondern war darüber hinaus mit einer zweiten widersprüchlichen Anforderung konfrontiert. Das Netzwerk musste die Nähe der Politik suchen und sich diese gleichzeitig vom Leibe halten: Das VSB-Netzwerk wurde primär ins Leben gerufen, um Pressionen der internationalen und nationalen Politik abfedern und mit den Bedürfnissen des Bankensektors kompati-

50 Vgl. etwa die Formen des Vertrags zugunsten Dritter (Art. 112 OR). Hierzu m.w.N. Patrick Krauskopf (2000) Der Vertrag zugunsten Dritter, Fribourg: Universitätsverlag.

51 So auch Urs Zulauf (1994) »Gläubigerschutz und Vertrauensschutz: Zur Sorgfaltspflicht der Bank im öffentlichen Recht der Schweiz«, 113 II Zeitschrift für Schweizerisches Recht (ZSR), 359-535, 434 ff.; vgl. Art. 5 f. KG.

52 Tatsächlich beseitigt die VSB, an welcher von Beginn weg die wichtigsten Banken der Schweiz beteiligt waren, offensichtlich den Wettbewerb im geregelten Bereich: So auch explizit Zulauf (Fn. 51). Nach der auf den Finanzmarkt zugeschnittenen politischen Ausnahmeklausel von Art. 6 Abs. 2 KG sind jedoch solche Wettbewerbsabreden zu rechtfertigen. Vgl. hierzu die Botschaft des Bundesrats zur Revision in Bundesblatt 1994, 564, die explizit auf den Bankensektor hinweist. 
bilisieren zu können. ${ }^{53}$ Entsprechend wurde von Beginn weg Anschluss an diese politische Dimension gesucht, zunächst durch die Einbindung der Nationalbank als Netzwerkzentrum und in der zweiten Phase der VSB durch die Integration der EBK als obere Aufsichtsinstanz des Netzwerks. Dennoch blieb es immer Ziel der VSB, den Einfluss der Politik möglichst »wirtschaftskompatibel « zu halten. ${ }^{54}$ Wie konnte nun die Wirtschaft mit dem Netzwerk auf die paradoxe Anforderung reagieren, die Politik einzubeziehen und sie sich gleichzeitig vom Leibe zu halten? Ich möchte hier zwei Dimensionen unterscheiden, erstens die Kommunikation über den Bedarf an kollektiv bindenden Entscheidungen und zweitens das Problem der Legitimation.

\section{a) Kommunikation über den Bedarf an kollektiv bindenden Entscheidungen}

Will die Wirtschaft der Politik den Bedarf (oder eben Nichtbedarf) an kollektiv bindenden Entscheidungen signalisieren, kann diese Kommunikation nicht in beliebiger Art und an beliebiger Stelle erfolgen. Nimmt man das Modell der polykontexturalen Gesellschaft ernst, dann gibt es aufgrund der unüberbrückbaren Autonomie der jeweiligen Systeme und aufgrund des entsprechenden babylonischen Sprachgewirrs keinen direkten Kommunikationskanal zwischen Wirtschaft und Politik, durch welchen diese Information ohne weiteres übermittelt werden könnten. Und entsprechend können auch im Netzwerk die verschiedenen Rationalitäten nicht derart »zueinander finden «, dass sie gemeinsam, wie etwa bei Savigny, im alles umfassenden Weltgeist aufgehen würden. ${ }^{55}$ Nach systemtheoretisch-evolutorischer Sichtweise bleibt der Wirtschaft keine andere Möglichkeit, als die Politik am Ort der strukturellen Kopplung derart zu irritieren, dass im System der Politik die erforderlichen Variationen ausgebildet und selegiert werden. Obwohl die Erfolgschancen, durch Irritationen bei einem Umweltsystem die gewünschten Selektionen zu erwirken, entsprechend gering zu veranschlagen sind, wurden mit dem Netzwerk VSB verhältnismäßig wirkungsvolle morphogene Strukturen unter Anschluss an mehreren strukturellen Kopplungen installiert:

- Während das Netzwerk dem Recht primär als ein Bündel von Verträgen erscheint, stellt es sich aufgrund des Netzwerkeffekts der Politik gleichzeitig als Organisation, genauer noch, als Kollektivakteur dar. Damit vermag der entsprechende Teilbereich der Wirtschaft der gesetzgebenden Politik als Identifikationspunkt zu erscheinen, an welchem die Politik Informationen über das andere System und vor allem die Bedingungen der Möglichkeiten kollektiv bindender Entscheide bezieht sowie die Bedingungen der Möglichkeiten zukünftiger kollektiv bindender Entscheide kommuniziert, um anhand der Reaktionen die Chancen der Realisierung politischer

53 Vgl. oben Kap. I.

54 Oben bei Fn. 43. Torsten Strulik (2000) „Governance globalisierter Finanzmärkte: PolicyNetzwerke und Kontextsteuerung im Bankensystem«, in: Jörg Sydow und Arnold Windeler (Hg.) Steuerung von Netzwerken, Opladen: Westdeutscher, 301-326, 322. Strulik weist darauf hin, dass analoges auch für die internationale Ebene der Selbstregulierung gilt. De Capitani weist darauf hin, dass die Banken mit der VSB 2003 versuchten, die forscher agierende VSB-Aufsichtskommission zurückzubinden: Capitani (Fn. 8) 64.

55 Friedrich Carl von Savigny (1840/1973) System des heutigen Römischen Rechts, Bd. 1-4, Berlin: Veit, Band I, $22 \mathrm{f}$. 
Programme zu testen. Die Wirtschaft ihrerseits liest diese Kommunikation über geplante kollektiv bindende Entscheide als Kosten ab und nutzt wiederum die Organisation, um den Bedarf oder Nichtbedarf an kollektiven Entscheidungen mitzuteilen. ${ }^{56}$ Für den Bankensektor ist diese Möglichkeit, der Politik den Nichtbedarf einer gesetzgeberischen Interventionen über Sorgfaltspflichten mitzuteilen, von zentraler Bedeutung. Denn auf diese Weise konnten und können der Politik die wirtschaftsinternen Bemühungen, ein punktuelles Equilibrium zur Politik zu erreichen, relativ schnell und verlässlich zu Kenntnis gebracht werden. In casu drängte denn auch die Politik auf eine Lösung der (politikinternen) Krise des Bankenplatzes Schweiz, und aus Sicht der Wirtschaft sollten die langsam mahlenden und (für die Wirtschaft) unberechenbaren Mühlen der Gesetzgebung möglichst gar nicht erst in Gang kommen. ${ }^{57}$

- Allein über die strukturelle Kopplung Vertrag wäre die gesetzgebende Politik zwar nicht zu erreichen gewesen. Allerdings wurde es dem Netzwerk mit der vertraglichen Einbindung der Nationalbank, eines politischen Akteurs mit großem Renommee, als Netzwerkzentrum gerade für den Beginn, kurz nach dem weitreichenden TexonSkandal erleichtert, von der gesetzgebenden Politik gehört zu werden. Denn über die strukturelle Kopplung Vertrag, mit welcher Projekte verschiedener Systeme mit der Hilfe von Recht auf eine bestimmte Dauer stabilisiert werden können, hatte die Nationalbank die Anliegen der Politik relativ direkt ins Netzwerk einbringen können. ${ }^{58} \mathrm{Ne}-$ ben dieser spezifischen »Entfesselung « eines schmalen Bereichs von Irritationen werden jedoch die Irritationen am Ort der strukturellen Kopplung gleichzeitig beschränkt und damit eine wichtige Voraussetzung zur Kompatibilisierung der Systeme geschaffen: Die Netzwerkteilnehmer geben ihre Autonomie in zweierlei Hinsicht auf, um die Kooperation im Netzwerk zu ermöglichen. Nicht nur enthalten sich die involvierten Wirtschaftsteilnehmer jenen Handlungen, die ihnen zwar kompetitive Vorteile auf dem Markt brächten, aber die Kooperation im Netzwerk in Frage stellten. Darüber hinaus reduzieren die am Netzwerk beteiligten Wirtschaftsteilnehmer ihre Autonomie in der wechselseitigen Gestaltung ihrer Geschäftsbeziehungen, ebenso wie sich die beteiligte staatliche Verwaltung in ihren Möglichkeiten einschränkt, auf dem Weg des hierarchischen Gesetzgebungsprozesses auf den Bankensektor einzuwirken. ${ }^{59}$

Diese Selbstbeschränkung der beteiligten Systeme setzt die Erhaltung der jeweiligen Eigenrationalität voraus. So zeigt das Beispiel der frühen VSB, dass sich das Netzwerk mit einer schwach legitimierten öffentlichen Institution - in casu der Nationalbank, die zwar über die erforderliche Reputation, aber über keine passende gesetzliche

56 André Brodocz (1996) »Strukturelle Kopplung durch Verbände«, 2 Soziale Systeme, 361387, 367; Gunther Teubner (1999) »Polykorporatismus: Der Staat als >Netzwerk < öffentlicher und privater Akteure«, in: Hauke Brunkhorst und Peter Niesen (Hg.) Das Recht der Republik, Frankfurt a.M.: Suhrkamp, 346-372, 349; Niklas Luhmann (2002) Die Politik der Gesellschaft, Frankfurt a.M.: Suhrkamp, 396 ff.

57 Vgl. hierzu den Leitartikel der NZZ vom 7./.8.5.77; vgl. auch oben Kap. I.

58 Zur strukturellen Kopplung Vertrag u.a. Gunther Teubner (2000) »Ein Fall von struktureller Korruption? Die Familienbürgschaft in der Kollision unverträglicher Handlungslogiken (BVerfGE 89, 214 ff.) «, 83 KritV, 388-404.

59 Vgl. Teubner (Fn. 56) 363 m.w.N. 
Grundlage verfügte - nicht stabilisieren ließ. ${ }^{60}$ Denn wenn sich das Netzwerk im Schnittpunkt von Wirtschaft und Politik positioniert, um die Rahmenbedingungen eines Marktes maßgeblich zu beeinflussen, dann ist es auf den Anschluss an Macht- und Legitimationsressourcen der Politik angewiesen. Darauf ist zurückzukommen.

Das Netzwerk stellt also eine institutionelle Strategie der sich fremd gewordenen Subsystemen der Gesellschaft dar, sich unter der Bedingung der Erhaltung der jeweiligen Eigenrationalität aufeinander einzulassen, um ein punktuelles Equilibrium zu suchen. Insofern kommt der chamäleonhaften Netzwerkform eine evolutorische Bedeutung in der Kompatibilisierung widersprüchlicher Rationalitäten zu. ${ }^{61}$ Im Überblick: Mithilfe der strukturellen Kopplungen tastet die Wirtschaft die eigene Position im Verhältnis zu den Anforderungen der Politik ab, um anhand dieser Information unter Ausnutzung der strukturellen Kopplung Vertrag Veränderungen im Teilbereich des Bankensektors auszulösen, die ihrerseits über die strukturelle Kopplung Organisation der Politik zur Kenntnis gebracht und auf ihren Erfolg hin überprüft werden usw. Diesen morphogenen Prozess gilt es aus evolutionstheoretischer Sicht nicht nur zu respektieren, sondern auch normativ abzusichern, womit sich eine rechtliche Rekonstruktion des Netzwerks allein als unzusammenhängendes Bündel von Verträgen oder allein als Organisation ebenso verbietet wie eine Rekonstruktion allein aus wirtschaftlicher oder politischer Sicht. Vielmehr gilt es erstens den Re-entry der Organisationsform in den Inhalt der Verträge derart abzusichern, dass der Prozess der Abgleichung zwischen den Systemen Wirtschaft und Politik am Ort der strukturellen Kopplung Organisation »spielen« kann. Und zweitens sind die jeweils unterschiedlichen Rationalitäten, die im Netzwerk aufeinander treffen, gegeneinander abzusichern. Zusammengefasst: Es geht um die institutionelle Absicherung des Netzwerks im Lichte seiner evolutorischen Funktion.

Doch das ist nur die eine Seite der Medaille. Die Vertragsform bietet - wie BGE $109 \mathrm{Ib} 149$ zeigt - auch einen guten Schutz gegen Ansprüche und Einmischungen Dritter: Das Netzwerk soll dem Recht gar nicht erst als Organisation erscheinen, womit Dritte an die einzelnen Netzwerkknoten verwiesen werden, welche sich jedoch gegen den Vorwurf der Rechtsverletzung einerseits auf die Bilateralität des Vertrags und andererseits auf das übergeordnete politische Moment des Netzwerkeffekts, d. h. die Marktordnung berufen. Damit eröffnet sich jedoch ein Legitimationsproblem des Netzwerks, auf das ich nun eingehen will.

\section{b) Legitimation}

Ganz ohne politische Beteiligung vermochte der Bankensektor in casu VSB von Beginn weg nicht auszukommen. Zunächst benötigten die Banken eine neutrale schlichtende Stelle, um die Konkurrenz überwinden und die Kooperation in Gang setzen zu können. ${ }^{62}$ Sodann verhalf die Beteiligung eines politischen Akteurs dem Netzwerk

60 Dies wurde bereits von Christian Schmid (1983) »Die neue Vereinbarung über die Sorgfaltspflichten der Banken«, 79 SJZ, 69-73, 72, moniert.

61 Grundlegend hierzu Teubner (Fn. 28) 570 ff.; Teubner (Fn. 2) $40 \mathrm{ff}$.

62 Tatsächlich nahm die Nationalbank bei der Bewältigung des Texon-Skandals eine führende Rolle ein: vgl. NZZ vom 27.4.77: 17 und vom 28.4.77: 13. 
dazu, von der Politik besser gehört zu werden. Aber nicht nur das: Um eine Marktordnung zu implementieren, benötigte die wirtschaftliche Rationalität die politischen Symbole des Zwangs und des Unverfügbaren. Was ist damit gemeint? Es liegt bereits im Begriff der Marktordnung angelegt, dass diese für einen ganzen Teilbereich der Wirtschaft gelten soll, folglich eine Vielzahl von Marktteilnehmern auf unterschiedlichen Ebenen betrifft und diese Marktteilnehmer in ihren wirtschaftlichen Möglichkeiten einschränkt, und dass die Marktordnung in letzter Instanz auch mit Zwang durchgesetzt werden soll. Wird aber Recht - das im Verbund mit dem politischen Zwangssymbol auftritt - instrumental eingesetzt, etwa um wie in casu eine bestimmte Marktordnung abzusichern, eröffnet sich die Frage der Legitimation dieses Rechts. Der erläuterte Fall BGE 109 Ib 146 verdeutlicht diese Problematik: Zur Debatte standen in BGE 109 Ib 146 die Interessen des Treuhänder-Verbands und dessen Mitglieder. Diese erlitten mit der verschärften VSB-Regelung einen beachtlichen Wettbewerbsnachteil, konnten jedoch auf die vor allem von den Banken und der Nationalbank erstellte VSB in keiner Weise Einfluss nehmen. Die Reaktionen auf BGE 109 Ib 146 zeigen denn auch, dass eine rein privatrechtliche Legitimation, die die rechtliche Stabilisierung bilateraler Differenzen mit dem freien Willen der Beteiligten und den Ausschluss von Drittinteressen mit dem Mechanismus des selbststeuernden Marktes legitimiert, auf Dauer zu schwach war: Im Netzwerk VSB ging es ja nicht nur um ein Bündel Verträge. Vielmehr ermöglichte der intendierte Netzwerkeffekt, erreicht durch die Verbindung überragender Marktmacht und unter Einbeziehung der Politik etwas, das weit über die Summe der einzelnen Verträge hinaus ging, nämlich eine umfassende Marktordnung - und zwar eines Schlüsselmarktes von zentraler politischer Bedeutung. Diese Problematik wird im heutigen Recht bereits dadurch signalisiert, dass das Kartellgesetz nur aufgrund politischer Ausnahmeklauseln keine Anwendung findet. ${ }^{63}$ Die Legitimationsproblematik wurde also durch die traditionellen privatrechtlichen Legitimationsmechanismen nicht gelöst, sondern es musste auf politische Legitimationsmechanismen zurückgegriffen werden. ${ }^{64}$

Zur Erlangung der Legitimation kann freilich die Wirtschaft nicht ein weitverzweigtes Beteiligungsverfahren nachbilden, wie es dem demokratischen Rechtsstaat zur Legitimationserzeugung dient, ohne die eigene Rationalität, die auf die Senkung der Transaktionskosten pocht, zu verleugnen. ${ }^{65}$ In dieser Beziehung unterliegt sie den systemeigenen Constraints. Die Wirtschaft muss folglich einen anderen Weg finden, um für ihre Selbstregulierung die politische Symbolik des Zwangs und vor allem der Legitimation zu erlangen.

Dies geschah im Beispiel der VSB zunächst damit, dass ein politischer Akteur durch Vertrag ins Netzwerk einbezogen wurde. Während das Recht den am Vertrag beteiligten Rationalitäten erlaubt, ihr Projekt in die Form des Rechts zu kleiden und so an die

63 Vgl. Fn. 52.

$64 \mathrm{Zu}$ diesem Unterschied vgl. bereits Max Weber (1980) Wirtschaft und Gesellschaft, Tübingen: Mohr, 123. Zur Legitimation siehe auch Roger Brownsword (2006) »Zum Konzept des Netzwerks im englischen Vertragsrecht«, in diesem Heft I.2.

65 Zur Legitimation im demokratischen Rechtsstaat vgl. Luhmann (Fn. 33) 416 ff. 
rechtsstaatlichen Legitimationsmechanismen anzuschließen, sollte die Beteiligung der Politik die demokratischen Legitimationsmomente vermitteln. ${ }^{66}$ Die kooperatistische Lösung mit der Nationalbank als Netzwerkzentrum erwies sich allerdings als nicht stabilisierungsfähig, wie die rechtswissenschaftliche Kritik auf BGE 109 Ib 146 anzeigt: Der Nationalbank fehlte es bereits an einer gesetzlichen Grundlage, um in diesem Bereich überhaupt tätig zu werden; trotz des großen politischen Prestiges der Nationalbank konnte damit die demokratische Symbolik nicht in ausreichendem Maß übertragen werden, was die Gefahr jener politischer Interventionen heraufbeschwor, die abgewendet werden sollte. Entsprechend wurden strukturelle Änderungen im Netzwerk ausgelöst, um dieser Gefahr zu begegnen. Die Nationalbank wurde im Netzwerk durch die politisch besser abgestützte EBK ersetzt.

Doch selbst mit dem gesetzgeberischen Auftrag, über den die EBK verfügt, wäre eine kooperatistische Verbindung von Wirtschaft und Politik durch Vertrag nur schwer zu stabilisieren gewesen. Denn die vertragliche Verbindung zwischen Politik und Wirtschaft droht die bisherigen Legitimationsmechanismen des demokratischen Rechtsstaats gerade aufzulösen: Soll sich die staatliche Verwaltung auf andere Gesellschaftsbereiche in kooperatistischer Weise einlassen können, muss sie über die entsprechende Freiheit verfügen, Ziele und Mittel der Kooperation auszuhandeln. Die Verwaltung bestimmt hier nicht nur über den Weg zum politischen Ziel, sondern immer mehr auch über das Ziel selbst, womit die formelle und die materielle Gesetzmäßigkeit der Verwaltung als (für die Wirtschaft zentrale) Garanten rechtsstaatlich gebändigter Sozialgestaltung und als (für die Gesellschaft im Allgemeinen) Mittler demokratischer Legitimation in der Form eines elaborierten Beteiligungsverfahrens in Frage gestellt sind. ${ }^{67}$ Entsprechend wurden im VSB-Netzwerk die vertraglichen Bindungen zwischen Politik und Wirtschaft aufgelöst. Der politische Akteur wurde im Netzwerkzentrum durch die bankeneigene Branchenorganisation ersetzt, während die Politik sich neu an der Peripherie des Netzwerks positionierte und die Rolle der staatlichen Oberaufsicht übernahm. ${ }^{68}$ Auf diese Weise wurde die Netzwerkregelung einerseits einer unproblematischen privatrechtlichen Standesregelung angenähert und andererseits einer öffentlichrechtlichen politischen Rahmenordnung unterworfen. ${ }^{69}$ Mit diesen strukturellen Anpassungen des Netzwerks wurde den rechtswissenschaftlichen Kritikern Recht gegeben, die das Legitimationsproblem durch die Ausdehnung des Politischen und die Unterdrückung der widersprüchlichen Anforderungen konfligierender Handlungsbereiche zu lösen suchten. ${ }^{70}$

66 Dies deutet auch Müller an, wenn er die Beteiligung der Nationalbank als »amtlichen Anstrich « für die VSB bezeichnet: Müller (Fn. 7) $350 \mathrm{f}$.

67 Jürgen Habermas (1992) Faktizität und Geltung: Beiträge zur Diskurstheorie des Rechts und des demokratischen Rechtsstaats, Frankfurt a.M.: Suhrkamp, 519 ff.; Eberhard Schmidt-Assmann (2001) »Das Recht der Verwaltungsverträge zwischen gesetzlicher Bindung und administrativer Gestaltung «, in: Walter Drenseck (Hg.) Festschrift für Heinrich Wilhelm Kruse zum 70. Geburtstag, Köln: O. Schmidt, 65-78.

68 Illustrativ zum Verhältnis der VSB-Aufsichtskommission und der EBK: Capitani (Fn. 7); hierzu auch bereits Zuberbühler (Fn. 8).

69 Oben Kap. I.

70 Vgl. vor allem Müller (Fn. 7) und Rhinow (Fn. 18); g.M. Marti (Fn. 25) 576; eine andere differenzierende Meinung vertritt Schefer (Fn. 25) $1139 \mathrm{f}$. 
Mit dem teilweisen Rückzug in die traditionellen Dichotomien von Öffentlich und Privat konnte das Netzwerk zwar die Gefahr der Politisierung durch direkte gesetzgeberische Interventionen oder durch die richterliche Qualifizierung als öffentliches Recht, womit die politisierten »öffentlichen Interessen« das Netzwerk der wirtschaftlichen Rationalität wohl entrissen hätten, bis zu einem gewissen Grad abwenden. Allerdings verlor die VSB mit dem Rückzug in traditionellere Gefilde der Selbstregulierung auch ein bedeutendes Stück ihrer Fähigkeit, sich den widersprüchlichen Anforderungen konfligierender Rationalitäten anzupassen; der direkte Anschluss der Wirtschaft an die Symbiose von Recht und Politik zur Erlangung des Legitimationssymbols durch eine vertragliche Verbindung mit der Politik wurde weitgehend gekappt und damit die Möglichkeit der Morphogenese eingeschränkt. Zwar blieben die morphogenen Strukturen insofern erhalten, als sich Wirtschaft und Politik weiterhin über den Bedarf an kollektiven Entscheidungen am Ort der strukturellen Kopplung Organisation austauschen (genauer: irritieren) konnten. Und mit der EBK konnte die Politik weiterhin vom brachenspezifischen Wissen der Banken, wie eine funktionierende Marktordnung zu verfassen ist, profitieren und gleichzeitig ihre politischen Anforderungen ins Netzwerk einfließen lassen - allerdings nicht mehr in der bisherigen differenzierten kooperatistischen Weise, sondern nur noch im Sinne eines ordre public. $^{71}$ Die Politik verlor somit Einfluss auf die Details der Branchenregelung, und der Wirtschaft fehlte es nun an der Möglichkeit, die Morphogenese so weit zu treiben, dass die politischen Anforderungen relativ direkt über die strukturelle Kopplung Vertrag in die VSB hätten einfließen können. An den heute zahlreichen einschlägigen Gesetzesnormen lässt sich denn auch das Scheitern des Experimentalprogramms VSB, zumindest in seiner ursprünglichen Radikalität, ablesen. ${ }^{72}$ Und auch wenn ein beachtlicher Kern an Selbstregulierung erhalten geblieben ist, bleibt dennoch festzuhalten, dass die heutige Selbstregulierung - wie BGE 125 IV 139 zeigt - nicht mehr viel weiter zu führen vermag als eine traditionelle Standesregulierung. ${ }^{73}$

Aus der Sicht einer evolutorischen Theorie, die sich das Modell einer polykontexturalen Gesellschaft zugrunde legt, ist diese Entwicklung der VSB zu bedauern. Denn das ursprüngliche VSB-Netzwerk hatte sich mit seinen morphogenen Strukturen als vielversprechende Strategie erwiesen, widersprüchliche und gar paradoxe Anforderungen im Schnittpunkt konfligierender Gesellschaftssysteme zu entfalten und hiermit einen wichtigen Beitrag zur Koevolution und Integration der sich fremd gewordenen Gesellschaftssysteme zu leisten - bei gleichzeitiger Aufrechterhaltung der jeweiligen Eigenrationalitäten. ${ }^{74}$ Damit sollen die Schwierigkeiten, die das Netzwerk VSB für die Politik aufgeworfen hat, keineswegs verdrängt werden. Allerdings erfolgte meines Erachtens der Rückzug in die traditionelle Dichotomie von Privat und Öffentlich allzu

71 Zur Beschreibung einer reflexiven Steuerung des Bankensektors vgl. Strulik (Fn. 54) $308 \mathrm{f}$.

$72 \mathrm{Vgl}$. nur die mittlerweile ausführlichen Regelungen im Bundesgesetz vom 10. Oktober 1997 zur Bekämpfung der Geldwäscherei im Finanzsektor (Geldwäschereigesetz) und der entsprechenden Verordnung der EBK vom 18. Dezember 2002 zur Verhinderung von Geldwäscherei (EBK-Geldwäschereiverordnung).

73 Oben bei Fn. 24.

74 Messner (Fn. 26) 58 ff. 
früh. Denn es bestehen durchaus Möglichkeiten, eine netzwerkartige Struktur, die insbesondere auf der strukturellen Kopplung Vertrag aufbaut, um die sich fremd gewordenen Systeme zu integrieren, mit dem Erfordernis der Legitimation zu versöhnen. Ich will diese Lösungsansätze kurz skizzieren.

Im Sinne der rechtsstaatlichen Legitimationsmechanismen kann ganz grundlegend und im Rahmen der traditionellen Grundrechtsanwendung die Wirkung politischer Kommunikation auf die wirtschaftliche Seite des Netzwerks grundrechtlich überprüft werden:

- Sofern die Verwaltung Privaten die Möglichkeit gibt, mit ihrem Eintritt in ein kooperatives Verhältnis zur Verwaltung direkt zu verhindern, dass ein befehlsförmiges Verfahren eröffnet wird, ist die Freiheit des Privaten nicht vorausgesetzt, sondern erst dadurch, dass der Private vor die Wahl »Vertrag oder Verfügung « gesetzt wird, kann er sich durch das von der Verwaltung verlangte Verhalten für die - von der Verwaltung vorgeformte - Freiheit entscheiden. Die im Privatrecht traditionell vorausgesetzte Freiheit (als Freiheit etwas zu tun oder nicht zu tun) ist hier von der Verwaltung verliehen. Åkerstrøm Andersen hat überzeugend darauf hingewiesen, dass vor dieser verliehenen Freiheit als Prämisse nur noch die nackte »Gewissensfreiheit « (freedom of thought) als Überrest der voraussetzungslosen Freiheit steht, die dem Menschen kraft seines Menschseins zukommt. ${ }^{75}$ Die durch die Verwaltung verliehene Freiheit entfernt sich damit weit von der kantischen Freiheit, welcher keine Ursache vorangestellt ist, welcher ein Wert für sich selbst zukommt und als solches grundsätzlich auch nicht von sinnlichen und gesellschaftlichen Bedürfnissen abhängig ist. ${ }^{76}$ Bei dieser durch die Verwaltung verliehenen Freiheit fehlt die legitimierende Funktion der Selbstorganisationsdelegation des Privatrechts fast vollständig, weshalb ein solches kooperatistisches Verhältnis von Privaten und staatlicher Verwaltung dem öffentlichen Recht und dessen Legitimationsmechanismen zuzuordnen ist, in welchem es traditionell um die Absicherung der Freiheitsbereiche der Gesellschaft vor dem Staat sowie die Legitimierung der freiheitsbeschränkenden Eingriffe durch das Konzept des demokratischen Rechtsstaats geht. ${ }^{77}$ Bei der VSB stand zwar eine Marktregelung durch die gesetzgebende Politik in Aussicht, jedoch nicht derart unmittelbar, dass die Freiheit der Banken in der Entscheidung, ob und wie sie eine Selbstregulierung aufbauen wollen, als vom Staat vermittelt angesehen werden müsste. Vielmehr zeigt das proaktive Verhalten der Banken im Laufe der Evolution der VSB, dass maßgebliche Impulse zur Selbstregulierung jeweils von der Privaten Seite ausgingen. ${ }^{78}$

- Selbst wenn eine Selbstregulierung - insbesondere nach dem Kriterium der Entscheidungsfreiheit - dem Privatrecht zugewiesen wird, ist zunächst zu fragen, ob der

75 Niels Andersen (2004) »The Contractualisation of the Citizen - on the transformation of obligation into freedom«, 10 Soziale Systeme, 273-291.

76 Immanuel Kant (1993) Kritik der reinen Vernunft, Hamburg: Meiner, $561 \mathrm{f}$.

77 Vgl. hierzu auch die liberale Extremposition von Wolfgang Zöllner (1996) »Regelungsspielräume im Schuldvertragsrecht«, 196 AcP, 1-36, $26 \mathrm{ff}$. und $35 \mathrm{f}$.

78 In die entgegengesetzte Richtung argumentiert Müller (Fn. 7) 350 f., der dann aber die Beteiligung der Nationalbank als »amtlichen Anstrich« für die VSB bezeichnet. 
staatliche Druck zur Selbstregulierung oder der Druck zur Kooperation gegen Grundrechte verstößt. Da für die private Selbstregulierung die freie Zustimmung konstitutiv ist, stellt eine Selbstregulierung, die durch staatliche Verwaltungseinheiten aufgedrängt wird, potenziell einen Grundrechtsverstoß dar, der sich entsprechend zu rechtfertigen hat. Dabei gilt es auch die graduelle Evolution jener Kooperationen und Selbstregulierungen im Auge zu behalten, die durchaus zu Beginn im Rahmen der Grundrechte bleiben, später jedoch die Schwelle zur Grundrechtsverletzung überschreiten kann. ${ }^{79}$ So wäre etwa das von der EBK ausgesprochene Verbot eines VSB-Formulars, mit welchem Treuhänder deklarierten konnten, der wirtschaftliche Berechtigte sei ihnen zwar bekannt, dieser werde aber nicht offen gelegt, entsprechend auf die Einhaltung der Grundrechte zu prüfen gewesen. ${ }^{80}$

- Sodann ist daran festzuhalten, dass die Politik dann nicht aus der Gewährleistungsverantwortung entlassen werden darf, wenn sie sich an einer Regulierung beteiligt oder darauf einwirkt. Folglich müssen im gemischten hybriden Netzwerk die politischen Kommunikationen der öffentlichrechtlichen Netzwerkknoten als Verfügung angefochten werden können, weshalb auch der Begründung des Verwaltungsentscheides höhere Priorität zukommen muss. ${ }^{81}$ Damit ist allerdings nicht gesagt, dass jede entsprechende Gerichtsentscheidung direkt auf das Netzwerk und die privatrechtlichen Verträge durchschlagen würde: Sofern das Netzwerk als Bündel privatrechtlicher Verträge zu qualifizieren ist, muss sich eine Durchbrechung der privatrechtlichen Rationalität, die grundsätzlich die Bipolarität der Vertragspartner unter Ausschluss von Drittinteressen stabilisiert, in besonderem Maß rechtfertigen. ${ }^{82}$ Immerhin kann neben der Möglichkeit, von den politischen Akteuren Schadersatz zu fordern, bei entsprechendem Urteil auch eine Pflicht der politischen Akteure entstehen, auf das Netzwerk einzuwirken. ${ }^{83}$

Als Erweiterung einer solchen monokausalen Anwendung der Grundrechte gegenüber der Politik ist durch das Recht von den beteiligten Systemen eine neuartige Anwendung der Grundrechte einzufordern: eine polykontexturale Anwendung der Grundrechte. Denn wenn der Politik die verfassungsmäßige Gestaltung der polykontexturalen Gesamtgesellschaft nicht mehr zugetraut und zugemutet werden kann, dann ist in der Konsequenz von jedem gesellschaftlichen Subsystem eine entsprechende Selbstbeschränkung einzufordern, die die juridische Entfesselung und gleichzeitig die Zügelung systemspezifischer Rationalität gegenüber innerer spontaner Ordnung und gegenüber anderen Gesellschaftssektoren zum Ziel hat. ${ }^{84}$ Diese Forderung kann dem

79 Udo Di Fabio (1997) »Verwaltung und Verwaltungsrecht zwischen gesellschaftlicher Selbstregulierung und staatlicher Steuerung «, 56 Veröffentlichungen der Vereinigung der deutschen Staatsrechtslehrer: Kontrolle der auswärtigen Gewalt, 235-277, 256 f. und 275.

80 Vgl. oben Fn. 23.

81 Niklas Luhmann (1983) Legitimation durch Verfahren, Frankfurt a.M.: Suhrkamp, 215 ff.

82 Hierzu im Detail Peter Gauch (2003) »Zuschlag und Verfügung - Ein Beitrag zum öffentlichen Vergaberecht«, in: Peter Hänni (Hg.) Mensch und Staat: Festgabe der Rechtswissenschaftlichen Fakultät der Universität Freiburg für Thomas Fleiner zum 65. Geburtstag, Freiburg i.Ue.: Universitätsverlag, 595-617.

83 Vgl. auch Di Fabio (Fn. 79) 270 ff.

84 Gunther Teubner (2003) »Globale Zivilverfassungen: Alternativen zur staatszentrierten Verfassungstheorie«, 63 ZaöRV, 1-28; Messner (Fn. 26). 
VSB-Netzwerk auf verschiedenen Wegen aufgetragen werden. Insbesondere sollte die EBK als Genehmigungsinstanz diese Selbstbeschränkung anlässlich der Genehmigung der VSB im Rahmen der Generalklausel des Bankengesetzes, die die Gewährleistung der einwandfreien Geschäftstätigkeit vorschreibt, einfordern. Und die Gerichte sollten eine solche Selbstbeschränkung der wirtschaftlichen Rationalität einfordern, wenn sie - wie in BGE 109 Ib 146 - mit entsprechenden Fällen befasst werden.

Eine derartige inhaltliche Selbstbeschränkung der Wirtschaft wird oft unter dem Titel demokratischer Legitimationsmechanismen diskutiert. Ich will - wiederum skizzenartig - darlegen, wie demokratische Legitimationsmechanismen für die vorliegende Problematik rekonfiguriert werden könnten. Nach Autoren wie Habermas oder Grimm kann neuartiges Verwaltungshandeln, das sich aus den traditionellen Strukturen des demokratischen Rechtsstaats löst, Legitimation vor allem dadurch erhalten, dass es den Rückgriff auf normative Gründe mit einer inneren Demokratisierung ergänzt. Diese sogenannt innere Demokratisierung kann sich - zusätzlich zum Rechtsschutz, der auf die neuen Formen anzupassen ist - je nach Bereich aus der Verwaltungsbeteiligung ergeben, d. h. der verwaltungsinternen Institutionalisierung von Ombudsformen, gerichtsanalogen Verfahren, Anhörungen und Veröffentlichungen, Entscheidungsteilnahme von Betroffenen oder von Stellvertretern und so weiter. ${ }^{85}$ Wie bereits im Konzept des Sozialstaates gilt also, dass der Einzelne, respektive der Adressat des Verwaltungshandelns in jenen Stand gesetzt werden muss, in welchem er seine Interessen ausbilden, wahrnehmen und in den Entscheidungsprozessen zur Geltung bringen kann.

Aus Sicht einer systemtheoretisch fundierten evolutionären Theorie ist hier allerdings Vorsicht geboten, denn das Polykontexturale der heutigen Gesellschaft, d. h. die Ausdifferenzierung der Gesellschaft in verschiedene, nach einer jeweils unterschiedlichen Eigenlogik funktionierende Subsysteme gilt es als Errungenschaft der Moderne dem heutigen Gesellschaftsmodell zugrunde zu legen. Ob in der Ausdifferenzierung der Gesellschaft bereits - wie bei Teubner - ein normativer Grundsatz liegt, ${ }^{86}$ oder ob der Beobachter diese Ausdifferenzierung seinem Gesellschaftsmodell zugrunde legen muss, um ein adäquates Bild zu erhalten, auf das er sich sodann ausrichten kann, kann hier offen bleiben. Denn schon indem die polykontexturale Gesellschaft als Gesellschaft des Rechts erkannt wird, können Gefahren wie sie unter dem Titel des regulatorischen Trilemmas beschrieben wurden und die bei der Umsetzung von Habermas Vorschlägen drohen, erkannt und vermieden werden. ${ }^{87}$ Im Sinne der evolutorischen Theorie ist also vor allzu direkten Eingriffen in komplexe evolutorisch ausgebildete Strukturen fremder Subsysteme zu warnen. Vielmehr muss es - aus einer evolutions-

85 Habermas (Fn. 67) 527 ff.; ähnlich auch Dieter Grimm (1991) Die Zukunft der Verfassung, Frankfurt a.M.: Suhrkamp, 4, 14 und Volker Schneider (2000) »Möglichkeiten und Grenzen der Demokratisierung von Netzwerken in der Politik«, in: Jörg Sydow und Arnold Windeler (Hg.) Steuerung von Netzwerken, Opladen, $342 \mathrm{f}$.

86 Teubner (Fn. 58); hier trifft sich Di Fabio mit Teubner: Di Fabio (Fn. 79) 262.

87 Gunther Teubner (1984) »Das regulatorische Trilemma: Zur Diskussion um post-instrumentale Rechtsmodelle«, Quaderni Fiorentini per la Storia del Pensiero Giuridico Moderno, 109-149. 
theoretischen Sichtweise - darum gehen, mit Recht die innersystemische Neuausrichtung von Funktion, Leistung und Reflexion derart zu unterstützen, dass das System möglichst optimal auf intensive, komplexe und gar widersprüchliche Umweltanforderungen reagieren kann, ohne seine Eigenrationalität zu gefährden.

Damit ist eine evolutionäre Legitimation angesprochen, auf die ich nun zum Schluss eingehen will. Zunächst ist allerdings mit Habermas am Befund festzuhalten, dass zahlreiche Formen der Kooperation zwischen Staat und Privaten wie in casu das frühe VSBNetzwerk die Anforderungen der Politik an eine legitime Regelung nicht erfüllen: Die Wirtschaft knüpft zwar an die Politik an, vollzieht aber innerhalb der konkreten Kooperation, soweit sie der wirtschaftlichen Rationalität zugeordnet wird, den Re-entry des Politischen, soweit er die Strukturen der politikeigenen Morphogenese abbildet, nicht oder in nur ungenügender Weise. Auch heute signalisieren die erwähnten steten Forderungen eines bedeutenden Teils der Rechtswissenschaft, der das Netzwerk noch stärker ins öffentliche Recht zurückführen will, dass es der VSB immer noch an Legitimation mangelt und damit der Bankensektor die politischen Anforderungen im Hinblick auf ein punktuelles Equilibrium noch nicht erfüllt hat. Wie können also diese Anforderungen der Politik gegenüber der Wirtschaft durch eine polykontexturalen Anwendung der Grundrechte unterstützt werden? In den bisherigen Ausführungen wurden die morphogenen Struktu$\mathrm{ren}^{88}$ kooperatistischer und insbesondere netzwerkartiger Arrangements als evolutionäre Strategie zur Anpassung an die polykontexturale Gesellschaft identifiziert, die es durch Recht zu erhalten gilt. Derartige morphogene Strukturen, die durch Recht zu unterstützen sind, erleichtern es also den verschiedenen Gesellschaftssystemen, sich auf einander einzulassen, wobei die innerhalb dieser Strukturen aufeinander prallenden Rationalitäten durch rekonfigurierte Grundrechte gegeneinander abzusichern sind. ${ }^{89}$

Die schwierige Frage ist nun, wie die unterschiedlichen Systeme, unterstützt durch Recht, in diesem Prozess dazu angestoßen werden können, die Ausdifferenzierung ihrer Umweltsysteme sowie deren wichtigste Anforderungen an ein punktuelles Equilibrium zu berücksichtigen, oder in casu VSB: wie einerseits die Politik dazu gebracht wird, trotz politischem Programm, das es umzusetzen gilt und trotz fortbestehender Gewährleistungsverantwortung die wirtschaftliche Freiheit auch innerhalb der Selbstregulierung zu respektieren (als Re-entry der Wirtschaft in die Politik), und wie andererseits die Wirtschaft dazu gebracht werden kann, auf die politischen Anforderungen an ein legitimes VSB-Netzwerk einzugehen (als Re-entry der Politik in die Rationalität der Wirtschaft) insbesondere wenn gesetzgeberische Eingriffe der Politik aus Gründen der Überforderung des Gesetzgebers am konkreten Gegenstand vermieden werden sollen und gleichzeitig auch das Recht in einer polykontexturalen Gesellschaft weder über Wissen noch Möglichkeiten verfügt, der Wirtschaft konkrete und wirksame Regelungen zu implantieren. ${ }^{90}$

88 Damit ist vor allem die Feedbackschlaufe zwischen den strukturellen Kopplungen und der systeminternen Selektion, die das System in seiner Umwelt neu positioniert, gemeint: oben Kap. II.

89 Hierzu Teubner (Fn. 39) und (Fn. 58).

90 Dies wird zuweilen vergessen, wenn die mittlerweile bekannten Konzepte eines prozeduralen oder reflexiven Rechts diskutiert werden: vgl. etwa Strulik (Fn. 54) 322 ff., mit einer interessanten Optik auf die reflexive Steuerung des internationalen Bankensektors. 
Über diese Unsicherheit um das richtige Recht kann ein prozeduraler Ansatz, wie er von Wiethölter begründet wurde, hinweghelfen, indem er - ähnlich wie Habermas fordert - die Integration der Gesellschaft des Rechts durch die Beteiligung der Gesellschaft an der Recht-Fertigung des Rechts immer wieder aufs Neue hervorzubringen verspricht und hiermit gleichzeitig eine neuartige Legitimation hervorbringt. ${ }^{91}$ In einer prozeduralen Methode müssen sich vor allem die Gerichte mehr als bisher auf die ausdifferenzierte polykontexturale Gesellschaft einlassen. Die Aufgabe, gesellschaftsadäquate Normen zu finden, darf nicht mehr allein mittels Privatautonomie an die Wirtschaft und mittels Gesetzgebung an die Politik delegiert werden. Wenn es nun, wie oben festgestellt, darum geht, die Möglichkeiten der sich fremd gewordenen Subsysteme der Gesellschaft zu fördern, sich gegenseitig wahrzunehmen und auf jeweilige Anforderungen der Umweltsysteme einzugehen, dann gilt es den Blick nicht nur auf die strukturellen Kopplungen zu lenken, die es den Systemen wie gesehen ermöglichen, sich als Umwelt des jeweils anderen Systems wahrzunehmen, sondern auch die Strukturen im Auge zu behalten, anhand welchen Systeme auf Anforderungen aus ihrer Umwelt unter Berücksichtigung ihrer Eigenrationalität reagieren. Werden diese morphogenen Strukturen vom einen System derart bedroht, dass andere Systeme ihre Operationen und Strukturen nicht mehr an die sich verändernde Umwelt anpassen können, soll das Recht diese morphogenen Strukturen schützen, d. h. primär durch eine gerichtlich festgesetzte Kontrahierungspflicht, wenn wie in BGE 109 Ib 146 politische Legitimationsanforderungen an eine durch die Wirtschaft erstellte Marktordnung aufgrund struktureller Mängel nicht einbezogen werden. Das ist weiter zu erläutern:

Dogmatisch können solche Entscheide, die eine Austarierung der systeminternen Relation von Funktion, Leistung und Reflexion fördern sollen und im Beispiel der VSB einen Re-entry der politischen Anforderungen in die wirtschaftliche Rationalität verlangen, in sogenannten Nischen angesetzt werden: ${ }^{92}$ Zunächst ist in der mit der beobachteten Rationalität gekoppelten Teilrechtsordnung, also in casu dem Vertragsrecht, nach möglichen Nischen zu suchen, in welchen sich Variationen mit kompatibilisierendem Inhalt bilden können. Zumeist ist eine solche Nische in der Form einer Vorbehaltsnorm zu finden, die im Sinne einer Signalnorm auf die Kollisionsproblematik hinweist. Solche Kollisionsnormen sind in der Regel offen gefasst, um die evolutorische Dimension der Koexistenz von Systemen erfassen zu können. Traditionell übernehmen im Vertragsrecht die Generalklauseln der guten Sitten (Art. 19 f. OR, Art. 27 f. ZGB), zu Treu und Glauben (Art. 2 ZGB) sowie zur Anwendung des Rechts (Art. 1 ZGB) diese Funktion. ${ }^{93}$ Entsprechende Nischen, mit welchen das Netzwerk VSB dazu

91 Grundlegend Rudolf Wiethölter (1982)»Materialization and Proceduralization in Modern Law «, in: Gunther Teubner (Hg.) Dilemmas of Law in the Welfare State, Berlin: De Gruyter, 221-249.

92 Dieses Modell wurde für das Wirtschaftsrecht entwickelt: Amstutz (Fn. 27) $326 \mathrm{ff}$.

93 Vgl. hierzu jüngst die Bemerkungen von Peter Gauch (2005) »Der Schätzer und die Dritten«, in: Eugen Bucher, Claus-Wilhelm Canaris, Heinrich Honsell und Thomas Koller (Hg.) Norm und Wirkung: Festschrift für Wolfgang Wiegand zum 65. Geburtstag, Bern: Stämpfli, 823-843. 
gebracht werden könnte, politische Anforderungen mit der Rationalität des wirtschaftlichen Teilbereichs zu kompatibilisieren, sind jedoch auch im Kartellrecht vorhanden. ${ }^{94}$ Sind Nische und Kollisionsnorm eruiert, gilt es sodann die teilrechtsspezifische Rationalität, welche die Konsistenz innerhalb der Teilordnungen herstellt, auszumachen. Nur so werden einerseits der Grund der Kollision und andererseits die äußere Form der Evolutionsfähigkeit erkennbar. Diese teilrechtsspezifische Rationalität ist anhand der tragenden Präzedenzfälle abzulesen (Empirie), denn sie ist nicht bereits zum Vornherein festgeschrieben, sondern wird in den Anwendungsfällen konstruiert. ${ }^{95}$

Mit einem solchen Entscheid soll jedoch nicht nur die morphogene Struktur im unter Beurteilung stehenden Fall geschützt werden, sondern gerade durch die grobe Struktur des Entscheides auch ein komplexer Prozess der »sozialen « Rechtssetzung im Zusammenspiel von Recht, Wissenschaft und den am Konflikt beteiligten Systemen ausgelöst werden, in welchem die beteiligten Systeme unter Anleitung der gerichtlichen Erwägungen neue Variationen generieren, diese von der Rechtswissenschaft auf ihre dogmatische Konsistenz überprüft werden, sodann allenfalls den Gerichten zur Selektion vorgelegt werden, möglicherweise wiederum zurückgewiesen werden, usw. - bis eine stabilisierungsfähige Selektion gefunden wird. Kurz: Es geht darum, den Konflikt an die beteiligten Systeme zurückzuweisen - freilich unter Hinweis auf die zu suchende Lösung.

Im Fall von BGE $109 \mathrm{Ib} 146$ bestand das Problem im Wesentlichen darin, dass über die strukturelle Kopplung Vertrag von Politik und Recht die Legitimation für die erarbeitete Marktordnung hätte bezogen werden sollen. Die konkrete Ausgestaltung der VSB am Ort der strukturellen Kopplung Vertrag krankte jedoch daran, dass kein durch politische Mechanismen ausreichend legitimierter Vertragspartner ins Netzwerk einbezogen wurde, also die beiden Systeme Wirtschaft und Politik in ungenügender Weise strukturell zusammengeführt wurden. Somit hätten die Netzwerkknoten zur Kontrahierung mit betroffenen Dritten gezwungen werden sollen, bis das Netzwerk seine Marktordnung mit einer genügend legitimierten politischen Instanz gekoppelt hätte oder (wahrscheinlicher) eine andere genügende Legitimationsquelle erschlossen hätte, was in der Folge zu einem situationsspezifischen Re-entry der politischen Legitimationsanforderungen in die wirtschaftliche Rationalität der VSB und damit zu einem Schritt in Richtung eines punktuellen Equilibriums geführt hätte. Denn mit einem normativen Schutz der strukturellen Kopplung von Wirtschaft und (gesetzgebender) Politik wird auch die Möglichkeit einer Koevolution der Systeme sichergestellt, in welcher sich die beiden Systeme mit ihren jeweiligen Anforderungen irritieren können. Im Fall von BGE 109 Ib 146 wären damit die am Netzwerk beteiligten Banken dazu verpflichtet gewesen, die Mitglieder des Treuhänder-Verbands bei den Offenlegungspflichten gleich zu behandeln, wie die privilegierten Rechtsanwälte und Mitglieder der Treuhand- und Revisionskammer. Mit einem einfachen »so nicht « und relativ vagen Anforderungen an die Dogmatik im Sinn der oben festgehaltenen normativen

94 Oben Fn. 54.

95 Zum Ganzen: Amstutz (Fn. 27) 326 ff. 
Anforderungen soll somit ein Prozess freigesetzt werden, mit welchem in prozeduraler Weise unter Ausnützung evolutorischer Strukturen empirisch abgestützte Lösungen gesucht werden, die den strengen normativen Anforderungen des Rechts gehorchen und gleichzeitig in jenen Rationalitäten, die den Konflikt an das Recht herangetragen haben, Beachtung finden.

Zum Schluss ist darauf hinzuweisen, dass mit diesem prozeduralen Verfahren die verschiedenen am Konflikt beteiligten Systeme zusammen mit Recht in evolutorischer Weise am Ort struktureller Kopplungen auf einander abgeglichen werden und damit eine Koevolution der Systeme sichergestellt wird, welche die Ausdifferenzierung der konfligierenden Systeme gegenseitig fördert - im Sinne von Konstitutionalisierung und Kompatibilisierung der jeweiligen Systeme hin zu ihren Umweltsystemen. ${ }^{96}$ Diese durch die Rechtsprechung ausgelöste prozedurale Rechtsfortbildung, die neuartigen Problemstellungen in der heutigen polykontexturalen Gesellschaft angemessen ist und Normativität in formeller Weise unter Ausnützung der strukturellen Kopplungen von Recht und anderen gesellschaftlichen Systemen findet, bewegt sich auffallend parallel zur festgestellten Funktion des Rechts, die morphogenen Strukturen zwischen den beteiligten Systemen zu schützen, damit sich die Systeme, in casu Politik und Wirtschaft gegenseitig wahrnehmen und in ihrer Ausdifferenzierung garantieren können. Oder anders gesagt: Die materielle normative Forderung nach Schutz morphogener Strukturen (samt struktureller Kopplungen und Ausdifferenzierung beteiligter Systeme) zwischen den konfligierenden gesellschaftlichen Subsystemen, um die Evolutionsfähigkeit (oder, wie Wiethölter es nennt: Entwicklungs-Dynamiken) sicherstellen zu können, lässt sich in der Form der beschriebenen prozeduralen und evolutorischen Rechtssprechungsmethode an den Orten der strukturellen Kopplungen zwischen Recht und den verschiedenen gesellschaftlichen Subsystemen gewinnen. In diesem Sinn beteiligt das Recht - ähnlich wie von demokratischen Legitimationstheorien gefordert - die Gesellschaft des Rechts an der Rechtsfindung und schützt die ausdifferenzierte Gesellschaft - ähnlich wie bei rechtsstaatlichen Legitimationstheorien - unter Bewahrung der zentralen rechtsstaatlichen Funktion der Gerichte.

96 Ähnlich Rudolf Wiethölter (2003) »Recht-Fertigungen eines Gesellschafts-Rechts«, in: Christian Joerges und Gunther Teubner (Hg.) Rechtsverfassungsrecht: Recht-Fertigung zwischen Privatrechtsdogmatik und Gesellschaftstheorie, Baden-Baden: Nomos, 13-21. 